\title{
Experimental Study of a High-Frequency Fluidic Valve Fuel Injector
}

\author{
Eric M. Braun, ${ }^{*}$ Thania S. Balcazar, ${ }^{\dagger}$ Donald R. Wilson, ${ }^{\ddagger}$ and Frank K. Lu ${ }^{\S}$ \\ University of Texas at Arlington, Arlington, Texas, 76019
}

Fuel injectors composed of an orifice connected to a small plenum cavity were mounted on a detonation tube. These fuel injectors, termed fluidic valves, utilize their geometry and a supply pressure to deliver fuel and contain no moving parts. Behavior of these fluidic valves is characterized in order to determine their feasibility for integration with high-frequency pulsed or rotating detonation wave engines. Fuel flow is initiated just prior to ignition of the detonation tube to understand the interaction between the fluidic valves and the wave. Parametric studies have been conducted with the type of fuel injected, the orifice diameter, and the plenum cavity pressure. Results indicate that the detonation wave pressure temporarily shuts off the fluidic valve supply, but the wave products can be quickly expelled by the fresh fuel supply to allow for refueling. The interruption time of the valve scales with the injection and detonation wave pressure ratios as well as a characteristic time.

\section{Nomenclature}

$A^{*} \quad$ area (choked flow condition)

CJ Chapman-Jouguet property

$f$ frequency

$L \quad$ characteristic length

$\dot{m} \quad$ mass flow rate

$p_{0} \quad$ initial detonation tube pressure

$p_{C J} \quad$ CJ detonation wave pressure

$p_{p} \quad$ steady-state plenum pressure

$p_{s} \quad$ supply pressure to fluidic valve

PDE pulsed detonation engine

RDE rotating detonation engine

$t_{\text {int }} \quad$ interruption time

$U \quad$ speed

$x \quad$ Cartesian coordinate along axial direction

$\phi \quad$ equivalence ratio

$\pi_{C J} \quad$ detonation wave pressure ratio $\left(p_{C J} / p_{0}\right)$

$\pi_{I} \quad$ injector pressure ratio $\left(p_{p} / p_{0}\right)$

$\tau \quad$ dimensionless interruption time

\footnotetext{
*Graduate Research Associate, Aerodynamics Research Center, Department of Mechanical and Aerospace Engineering, Box 19018. Student Member.

$\dagger$ Undergraduate Research Assistant, Aerodynamics Research Center, Department of Mechanical and Aerospace Engineering, Box 19018. Student Member.

$\ddagger$ Professor, Aerodynamics Research Center, Department of Mechanical and Aerospace Engineering, Box 19018. Associate Fellow.

§Professor and Director, Aerodynamics Research Center, Department of Mechanical and Aerospace Engineering, Box 19018. Associate Fellow.
} 


\section{Introduction}

LTHOUGH pulsed fuel injection with mechanical valves has been successfully demonstrated with several A different concepts for pulsed detonation engines (PDEs), ${ }^{1,2}$ cycle frequencies hardly exceed $100 \mathrm{~Hz}$ in a single tube. The cycle frequency limit can be due to the valve itself or the required refilling time of the tube. For airbreathing PDEs where stable inlet flow must be maintained during flight, corresponding cycle frequencies will require state-of-the-art valves. A related and less developed engine concept, the rotating detonation wave engine (RDE), employs one or more detonation waves rotating circumferentially inside an annulus placed perpendicular to the inlet flow. ${ }^{3}$ Many recent experimental and computational studies have been conducted to assess its feasibility. ${ }^{4-11}$ Considering the angular speed of the rotating detonation wave, the effective operating frequency of the engine is $1-10 \mathrm{kHz}$. Hence, it has also been termed a continuous detonation engine since its inlet and nozzle flow will be quasi-steady. This frequency becomes important for satisfying flow requirements with side-mounted fuel injectors which certainly must be able to cycle beyond mechanical valve limits. Although an air-breathing RDE has yet to be experimentally tested, it can be expected that the air should enter the annulus from an axial direction to minimize flow losses. Presumably, a novel design of fuel jets impinging on the airflow can be developed to create sufficient mixing on a timescale between detonation wave fronts. However, the detonation wave will pass over the injector orifices and allow the high-pressure products to disrupt the steady flow. This backflow condition has been shown to occur during early RDE tests, ${ }^{12,13}$ and it can completely shut off the fuel supply if it lasts longer than the rotational period. The presence of multiple waves can further exacerbate this problem by reducing the time between each pressure front. Minimizing the backflow condition so as to return to steady-state injector flow is critical for a successful RDE.

Figure 1 shows a separate fuel-oxygen injector design by Nicholls and Cullen for a rocket-mode RDE tested in the $1960 \mathrm{~s}^{12}$ The impinging injector design and $0.5 \mathrm{~mm}$ orifice diameters are similar to rocket injector manifolds, ${ }^{14}$ and each orifice is followed by a plenum cavity. This design may be considered a fluidic valve. Unfortunately, no results were gathered to show that this specific geometry was effective since the RDE could not be started properly. This design of impinging injectors located on the wall normal to the detonation wave was also employed in early work by Bykovskii and Mitrofanov. ${ }^{15,16}$ Bykovskii and co-authors have published studies of many other injection attempts, summarized in a comprehensive review of RDE testing. ${ }^{3}$ Computational models typically assume an injection wall is present with a large number of orifices or micronozzles where refueling is temporarily blocked because the detonation wave pressure is larger than the supply pressure. ${ }^{9,10}$

Due to their potential for operation at the frequency required by an RDE, an experimental investigation of fluidic valves has been conducted. It is believed that fluidic valve characteristics such as overall geometry, orientation with respect to the annulus flow, backflow time, and required back pressure can be optimized using results from experimental and computational studies. The fluidic valve requires a small plenum

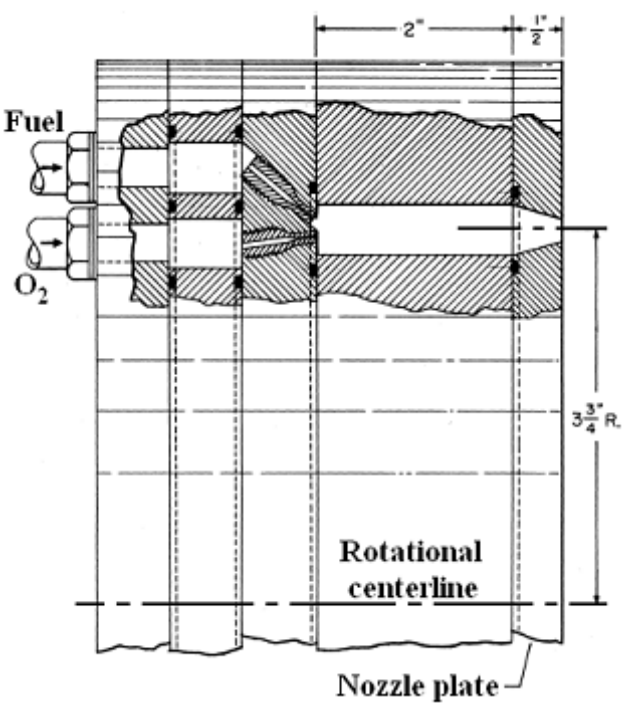

Figure 1. Impinging fuel-oxygen injection and mixing design from an early RDE study by Nicholls et al. ${ }^{12}$ cavity placed beneath the injector orifice which helps to act against combustion products entering the valve after each revolution of the detonation wave. Specifically, the contact surface separating detonation products and fresh propellant enters the valve but is quickly pushed out.

The first goal for these fluidic valve experiments is to understand how they respond to the detonation wave and if the fuel or oxygen behaves differently when injected separately. The orifice geometry and plenum pressure $p_{p}$ are then varied in an effort to optimize the fluidic valve effect. In the fluidic valve operating cycle where a plenum cavity is present, it is assumed that an incident shock and contact surface will enter as the detonation wave passes by. The incident shock will reflect off the walls of the cavity until the pressure behind the detonation front reduces enough to allow the products to exit from the cavity and allow for refueling to begin. The time in which the injector flow is shut off needs to be minimized. 


\section{Experimental Setup}

A schematic of the test apparatus appears in Fig. 2. A pair of radially-opposed fluidic valves labeled FV1 and FV2 was installed on a linear detonation tube for these experiments. The inner diameter of the detonation tube is $5.08 \mathrm{~cm}$ and the total length is $3.05 \mathrm{~m}$. The tube wall is $0.64 \mathrm{~cm}$ thick and the material is stainless steel. Table 1 lists the axial positions of the injectors, spark plug, and transducers relative to the left end of the tube in Fig. 2. Although the fluidic valves and pressure transducers are shown in the same plane in this two-dimensional figure, the valves were actually placed perpendicularly to the transducers on the circumference of the tube. PCB pressure transducers (model 111A24), rated for $7000 \mathrm{kPa}$, were used. The tube was evacuated to a pressure of about 10 torr before the fuel and oxygen were added. Static pressure transducers (Omega model PX209-060A5V) were mounted in the fill lines to record partial pressures for equivalence ratio calculations. An automotive spark plug (Bosch model Platinum +2 ) was flush-mounted in the center of the closed end of the tube. A mylar diaphragm with a thickness of $100 \mu \mathrm{m}$ was placed between the end of the tube and the exhaust tank.

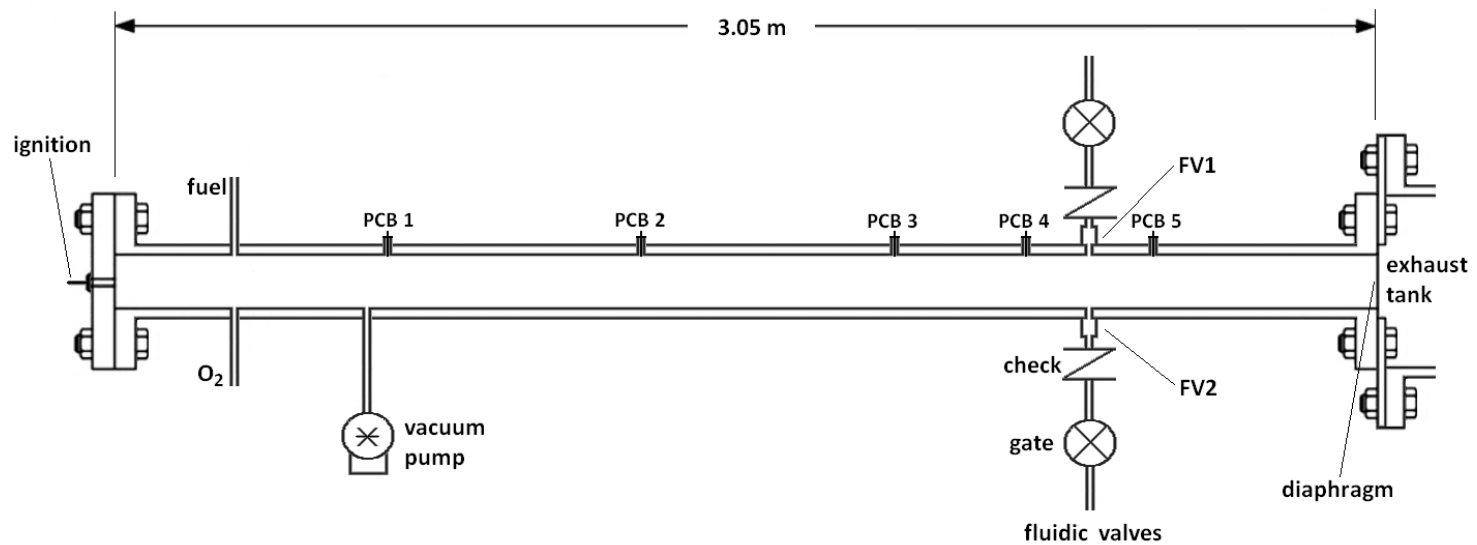

Figure 2. Schematic of the linear detonation tube.

As shown in the schematic, the fluidic valve begins with an orifice mounted on the wall of the tube. The orifice leads to a plenum cavity that operates at constant pressure. Upstream of the cavity, the cross-sectional area is again reduced and connected to a high-pressure fitting. The fluidic valve therefore has no moving parts. A computer-controlled, pneumatic gate valve was connected to each in order to initiate fueling into the detonation tube just prior to ignition. Check valves were also used to prevent a flashback from protruding through the cavity into the supply lines. The detailed geometry of the fluidic valve cavities is shown in Fig. $3(\mathrm{a})$. The axial coordinate system in the figure is relative to the surface of the detonation tube. The diameter of the injector orifice was made variable as part of the parametric studies to be conducted. The dimensions of the square cavity were fixed due to the size required for flush-mounted PCB pressure transducers (labeled INJ1 and INJ2). The circles shown in the middle of the cavity represent the diameter of the transducer faces. These transducers allow for the wave dynamics in the cavity to be tracked.

In each test, ignition and the operation of the fluidic valve were timed so the detonation wave would pass over the injector orifice immediately after the gas pressure had reached steady state. The detonation wave needed to be initiated immediately after the steady-state pressure was reached so the overall pressure in the tube was not significantly increased. Generally, a steady state was reached within $50 \mathrm{~ms}$ and the pressure rise measured in the tube was minimal. It is important to note that the steady-state injector cavity pressure is not a stagnation pressure due to the gas motion within. Consequently, the cavity pressure will rise if the orifice flow is interrupted by the passage of the detonation wave.

Three sets of injectors were machined to connect with the square cavity and detonation tube surface. Two sets of injectors consisted of a single hole with a diameter of $0.64 \mathrm{~cm}(0.25 \mathrm{in})$ or $0.32 \mathrm{~cm}(0.125 \mathrm{in})$. These diameters are larger than what has been used for previous RDE studies, and the $0.64 \mathrm{~cm}$ diameter hole resembles operation with almost no reduction in orifice area relative to the cavity. The third injector design consisted of an array of 25 evenly-spaced, $0.5 \mathrm{~mm}$ diameter holes all positioned normally to the detonation tube surface. For airbreathing RDEs, there may be a benefit if the injector diameters could be larger to reduce both flow losses and machining expenses. However, large diameters may also create stronger transverse 
Table 1. Axial positions of the linear detonation tube components.

\begin{tabular}{cc}
\hline Component & Axial distance $(\mathrm{cm})$ \\
\hline Spark plug & 0 \\
PCB 1 & 52.4 \\
PCB 2 & 113.2 \\
PCB 3 & 174.2 \\
PCB 4 & 204.7 \\
FV1, FV2 & 219.9 \\
PCB 5 & 235.2 \\
End; diaphragm & 305.0 \\
\hline
\end{tabular}

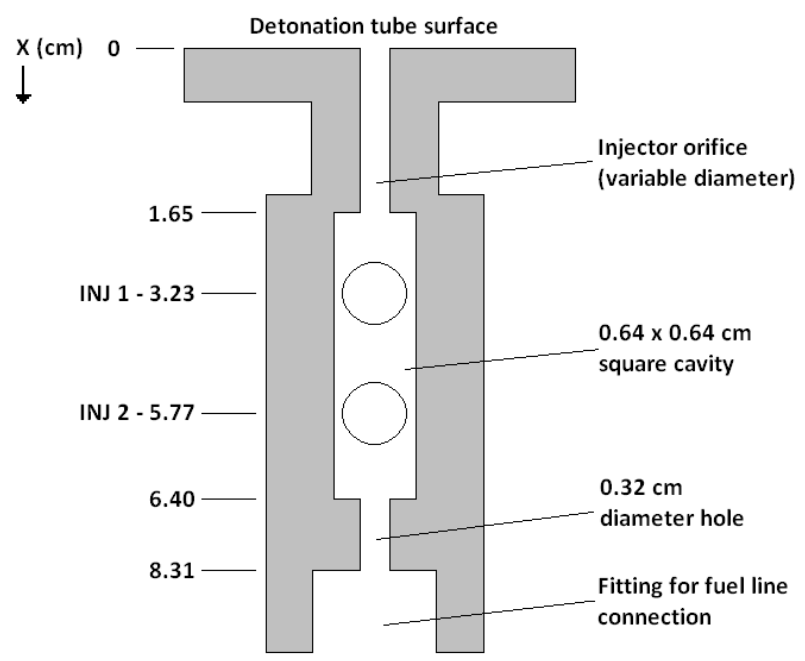

(a) Geometry of the fluidic valve cavities.

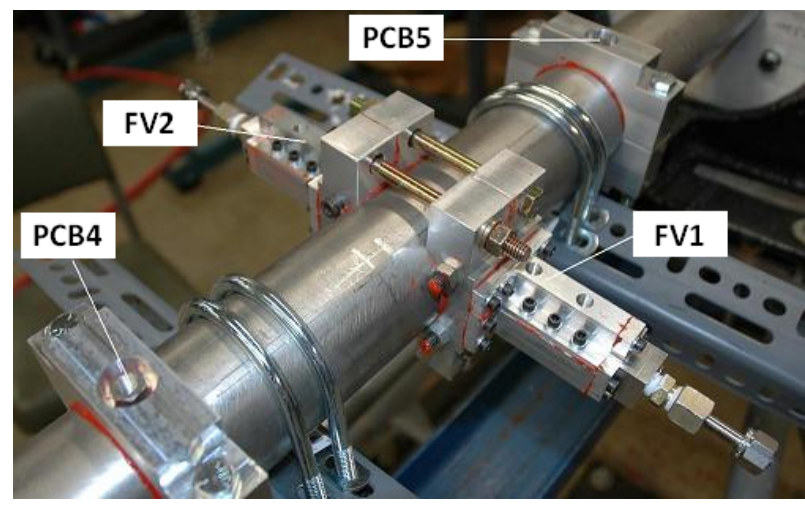

(c) Fluidic valves before instrumentation.

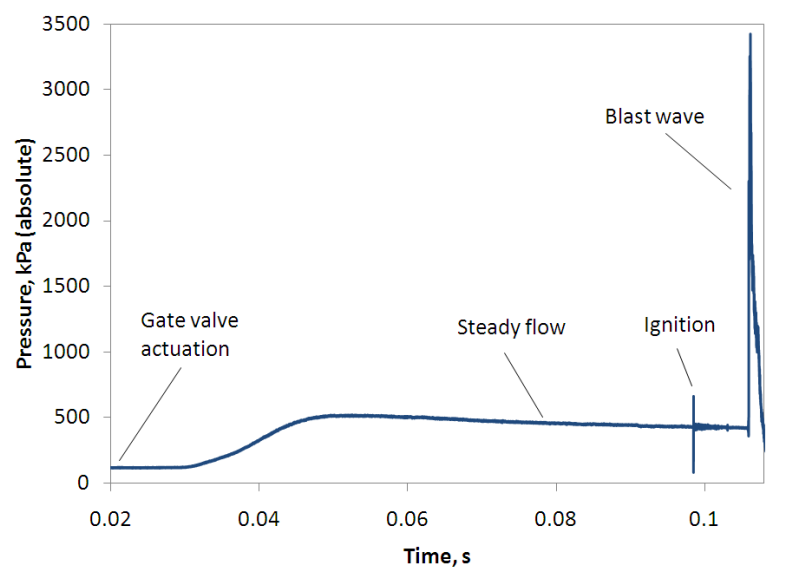

(b) Pressure trace from a transducer mounted in the fluidic valve showing timing.

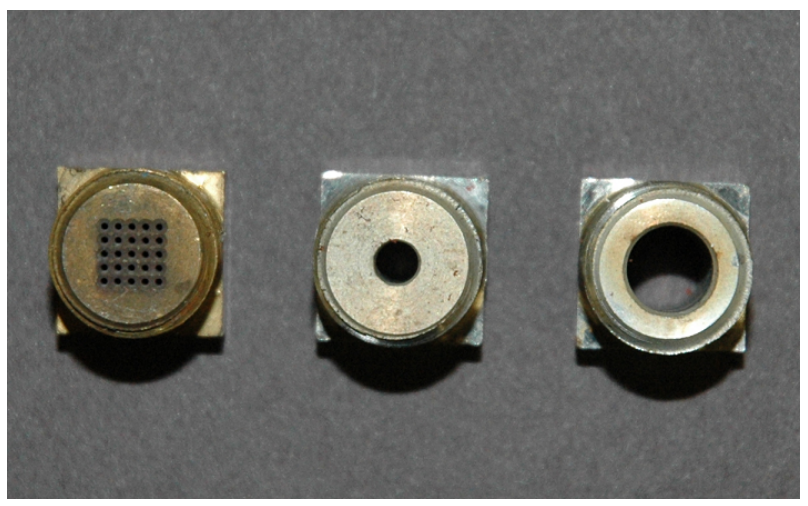

(d) Injector orifices.

Figure 3. Fluidic valve photographs and operational details. 
waves or allow the detonation wave to propagate into the cavity. Figures 3(c) and 3(d) show photographs of the valves and injector orifices. Note that Fig. 3(c) shows the injectors mounted perpendicularly from the pressure transducers.

\section{Testing}

\section{A. Propane/Oxygen Mixture with Argon Injection}

The fluidic valves were initially tested using all three injector designs to explore the effects of cavity pressure. The initial tube pressure of the stoichiometric propane/oxygen mixture was fixed at $120 \mathrm{kPa}$. Argon was supplied to the valve to simulate hydrocarbon fuels because of its comparable molecular weight. An inert gas with no chance of combustion was considered useful as a baseline. The first tests were run with the 0.32 $\mathrm{cm}$ diameter orifice and are summarized in Figs. 4(a)-4(d). Figure 4(a) shows a typical pressure reading from the two transducers in the square cavity shown in Fig. 3(a). In the figure, the cavity pressure is 620 $\mathrm{kPa}$. The transducers indicated a shock wave traveling down the square channel before reflecting off the end. The reflection created the $4100 \mathrm{kPa}$ pressure spike. Subsequent reflections were also measured until the pressure began to decrease at $0.105 \mathrm{~s}$. The pressure then was steady for about 0.5 ms before a rarefaction wave appeared as the flow began to again move out of the fluidic valve.

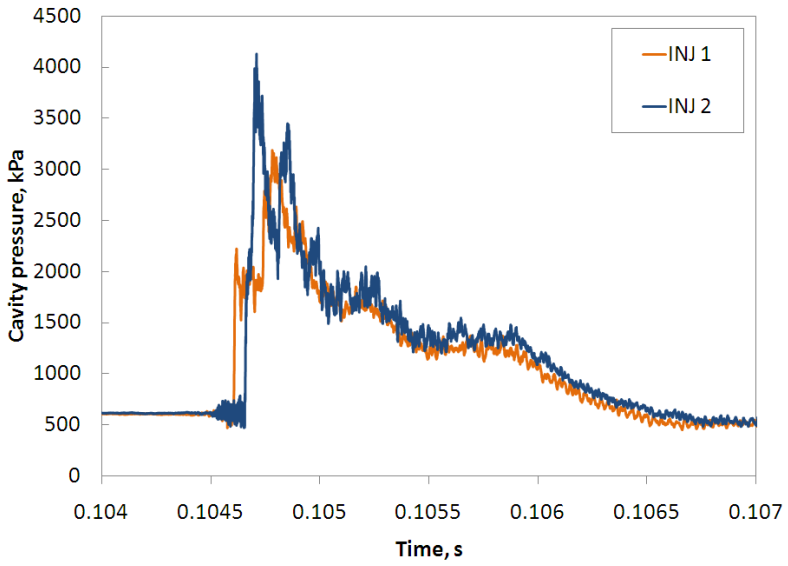

(a) Typical pressure readings inside the fluidic valve.

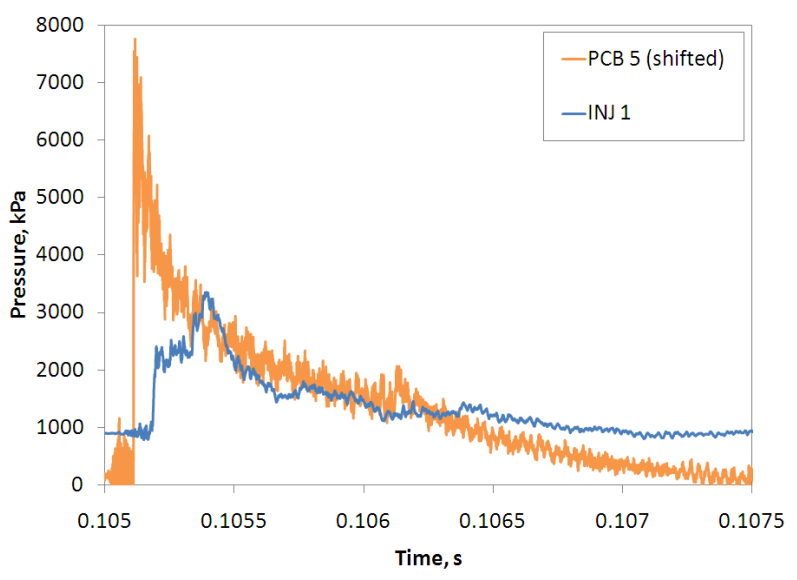

(c) Pressure trace with $p_{p}=960 \mathrm{kPa}$.

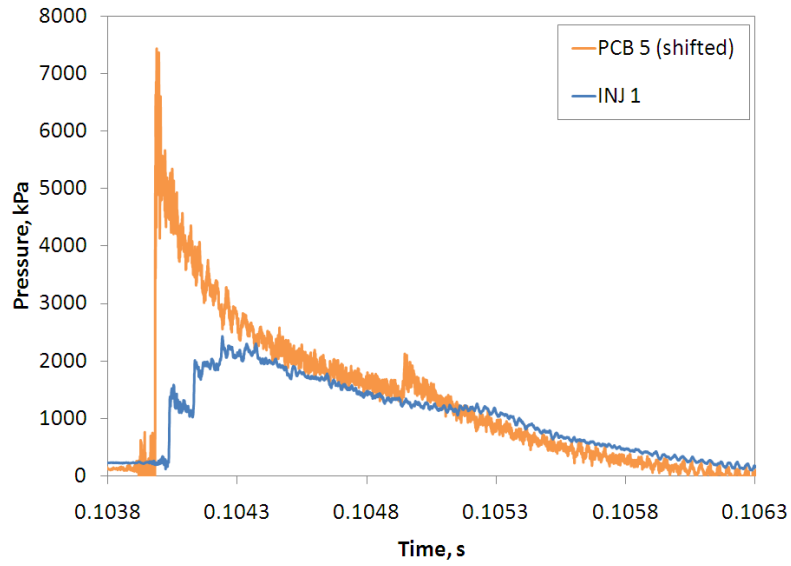

(b) Pressure trace with $p_{p}=230 \mathrm{kPa}$.

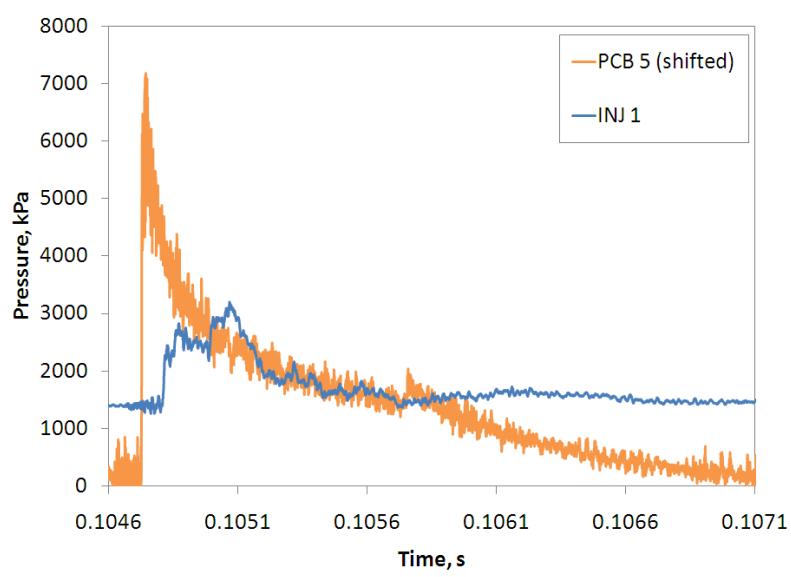

(d) Pressure trace with $p_{p}=1400 \mathrm{kPa}$.

Figure 4. Argon- $\mathrm{C}_{3} \mathrm{H}_{8} / \mathrm{O}_{2}$ injector-detonation wave interaction results $(0.32 \mathrm{~cm}$ orifice).

Figures 4(b)-4(d) depict the variation in the valve/detonation wave interaction as the steady-state injection pressure was increased. Since a comparison of pressure traces in the tube and cavity is desirable, the data from the transducer downstream of the injector (PCB 5) was time shifted to match with the detonation 
wave over the orifice. Hence there is a delay between the detonation wave front and the shock wave front in the cavity because of the distance from the orifice to the first cavity transducer. During this interaction, the chief concern is the time taken for the injector pressure to return a steady state and the mechanisms involved. For a cavity pressure about twice that of the initial tube pressure (Fig. 4(b)), it appears that multiple reflections occurred between 0.104-0.1043 s until the pressure became equivalent with the main tube. Afterwards, a rarefaction wave propagates through the cavity when the orifice flow becomes choked again as the cavity reaches steady state conditions.

If the steady-state injection pressure was raised, the time required to reach that pressure again dropped. For example, the steady state in Fig. 4(d) is reached again in about half the time as Fig. 4(b). This behavior inherently depends on the exponential decay of the rarefaction wave where reaching lower pressures takes an increasingly long time. Note the presence of a small spike in the rarefaction wave pressure in the middle of the detonation wave trace. This stems from a reflected shock in the tube as the diaphragm bursts and is not related to the fluidic valve actuation.

The $0.64 \mathrm{~cm}$ diameter orifice injector was designed to investigate the effect of the cavity geometry. The geometry with this orifice does not have a plenum cavity, but uses the wall at $x=6.40 \mathrm{~cm}$ (Fig. 3(a)) to establish a reflected shock. Despite an argon cylinder supply pressure of $4000 \mathrm{kPa}$, maintaining a pressure above that of the tube in this design is not possible. Figure 5(a) shows pressure traces from both transducers in the cavity, and they show a relatively long period of oscillations after the shock reflection. Note the wave front measured by the first transducer in Fig. 5(a) had a much higher pressure compared to Fig. 4(a). The wave speed was also increased to about $700 \mathrm{~m} / \mathrm{s}$. Figure $5(\mathrm{~b})$ shows that the cavity flow still scales with the detonation wave, but the pressure oscillations create doubt that all of the combustion products had left the cavity in time.

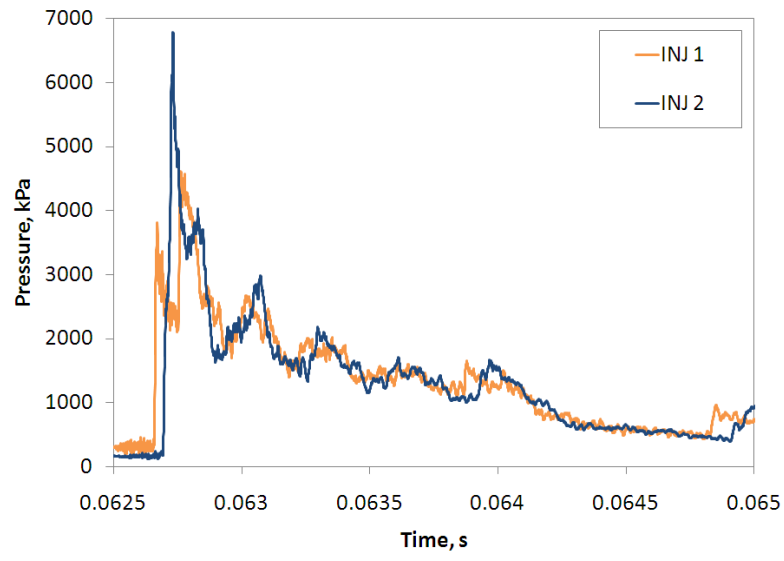

(a) Fluidic valve pressure readings from the transducers.

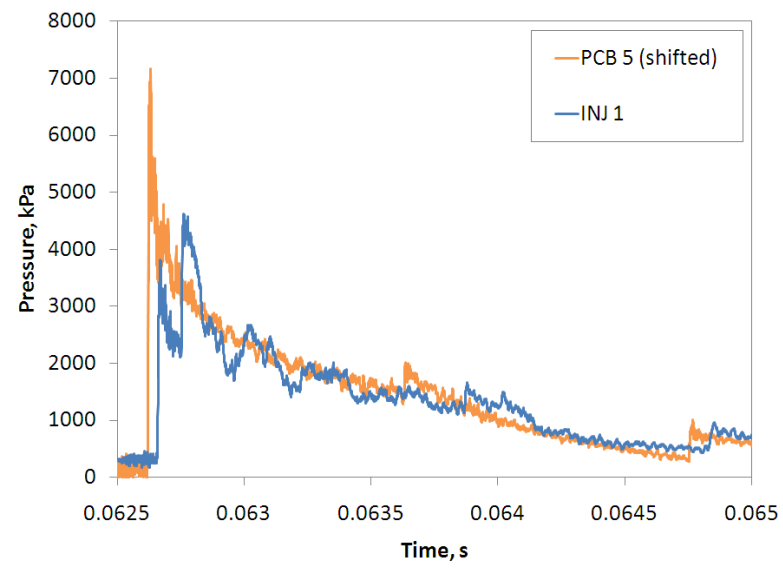

(b) Case with $310 \mathrm{kPa}$ steady state injection pressure.

Figure 5. Argon- $\mathrm{C}_{3} \mathrm{H}_{8} / \mathrm{O}_{2}$ injector-detonation wave interaction results with $0.64 \mathrm{~cm}$ orifice.

Figures 6(a)-6(d) chart the interaction pressure traces again for a variety of steady-state cavity pressures and the $25 \times 0.5 \mathrm{~mm}$ orifice. The combined orifice area with this array of holes is 61 percent of the single $0.32 \mathrm{~cm}$ hole. Figures 6(a) and 6(b) show that multiple reflections occurred in the cavity with this orifice geometry. Increasing the cavity pressure reduces the number of shock reflections. This cavity geometry was able to hold a plenum pressure higher than the other two injectors, and the case graphed in Fig. 6(d) shows the effect was to greatly decrease the backflow time. To create a figure of merit for comparison, the time between the detonation wave front and when the cavity pressure recovers to steady state is now referred to as the interruption time. Using pressure measurements alone, the calculated interruption time is not necessarily the backflow time and cannot be used to predict if the steady-state flow is indeed comprised of the detonation products or new propellants. However, it is useful for scaling and comparison purposes as will be shown later. When the $0.32 \mathrm{~cm}$ orifice is compared with this design, little difference in the interaction is seen when the steady state pressures are similar (e.g., Figs. 4(d) and 6(c)). The cylinder supply pressure for the Fig. 6(c) case is about $60 \%$ of what was used for the case in Fig. 4(d), even though the steady state injection pressures are similar. A mass flow rate comparison can be made using the relation $\dot{m} \propto A^{*} p$. When the mass flow rate per area of the different orifice diameters are compared as a function of supply pressure, 


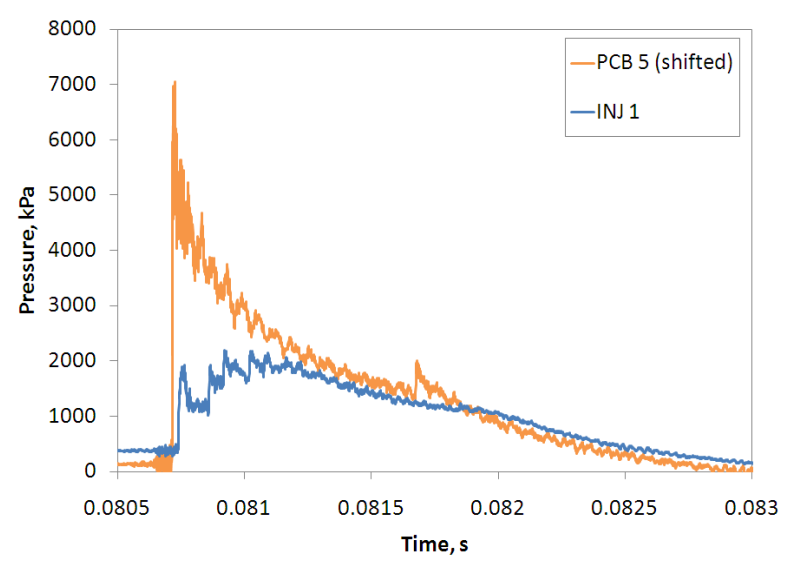

(a) $380 \mathrm{kPa}$ steady-state injection pressure.

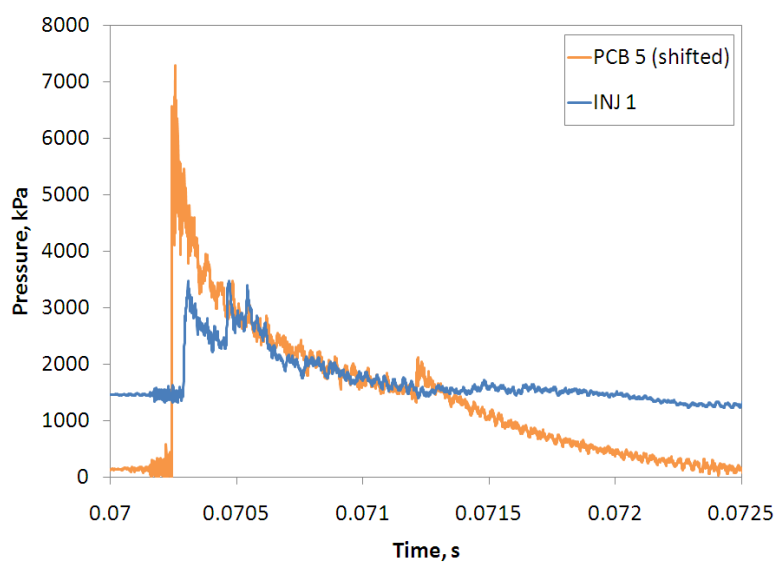

(c) $1500 \mathrm{kPa}$ steady-state injection pressure.

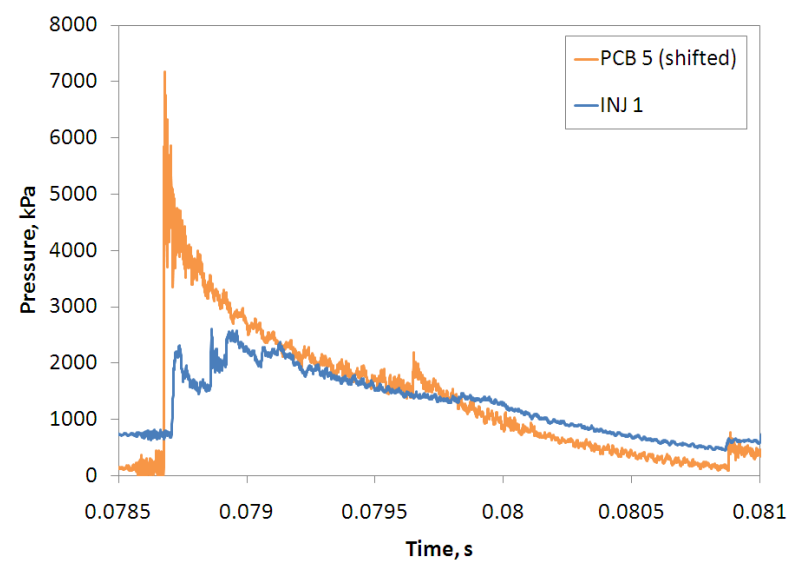

(b) $720 \mathrm{kPa}$ steady-state injection pressure.

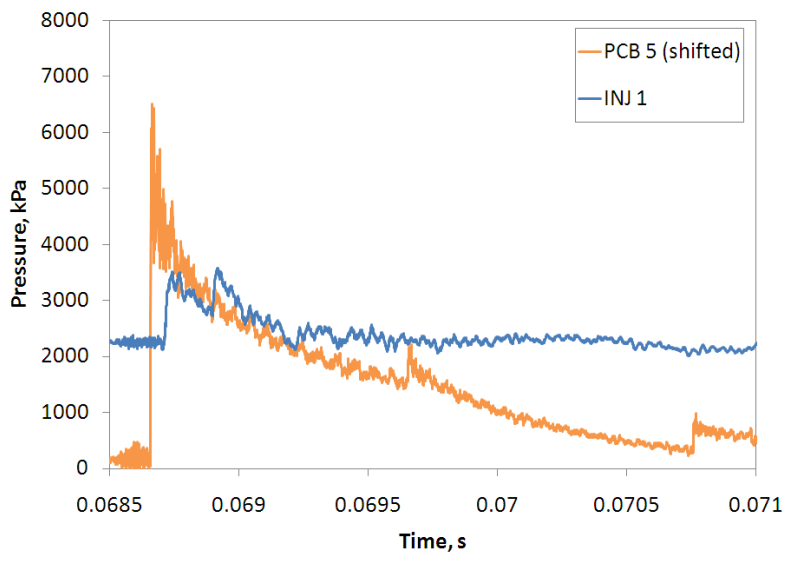

(d) $2300 \mathrm{kPa}$ steady-state injection pressure.

Figure 6. Argon $-\mathrm{C}_{3} \mathrm{H}_{8} / \mathrm{O}_{2}$ injector-detonation wave interaction results with $25 \times 0.5 \mathrm{~mm}$ orifice.

the results are similar. Therefore, designing a valve around a certain interruption time requires a tradeoff between mass flow rate though the orifice and supply pressure.

\section{B. Hydrogen/Oxygen Mixture with Separate Hydrogen and Oxygen Injection}

Tests using an initial hydrogen/oxygen tube mixture with separate injection of hydrogen and oxygen through the fluidic valves were conducted for a better understanding of scaling with different gases. From Fig. 7(a), it is apparent that the speed of the blast wave traveling in the fluidic valve is much faster when hydrogen fuel was used. The use of hydrogen also caused numerous shock reflections within the fluidic valve cavity. The oxygen fluidic valve behaved similar to when argon was used since the sound speeds of the two gases are similar. When the oxygen and hydrogen steady-state injection pressures are equivalent, Fig. 7(b) shows that the interruption times are similar. The observation that the interruption time scales with the detonation wave pressure is important since it shows that the properties of the gas injected do not apparently play a significant role.

\section{Analysis}

Much of the pressure trace information presented in the previous graphs was qualitative. However, $x-t$ diagrams can be constructed to show the flow characteristics in the plenum cavity. 


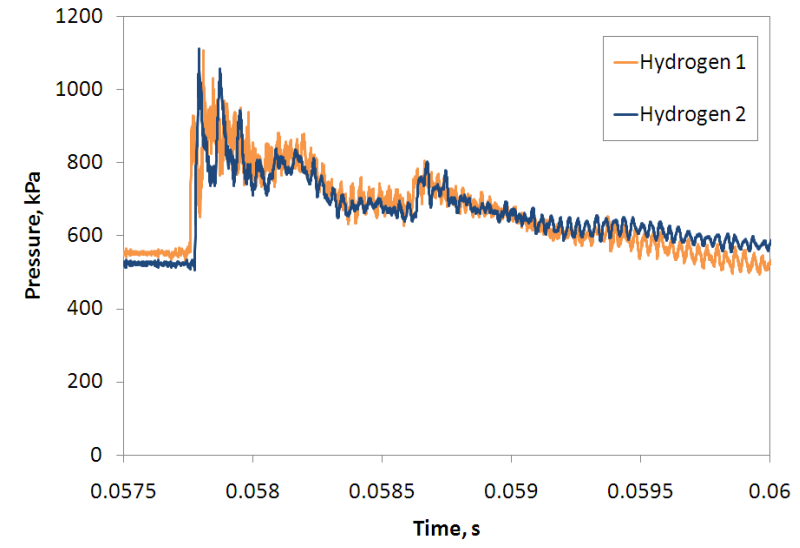

(a) Example of hydrogen pressure readings from the transducers mounted in the fluidic valve.

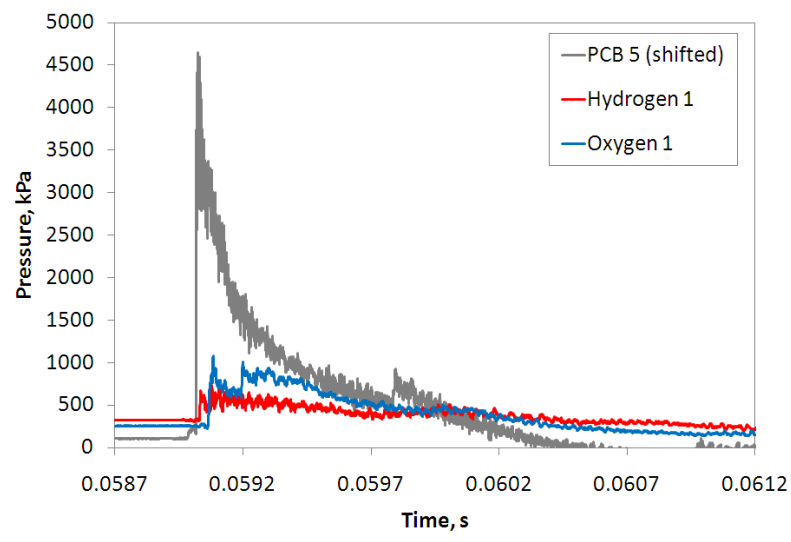

(b) $\mathrm{H}_{2}-\mathrm{O}_{2}$ injector-detonation wave interaction pressure trace with $310 / 275 \mathrm{kPa}$ steady-state injection pressure.

Figure 7. $\mathrm{H}_{2}-\mathrm{O}_{2}$ test results with the $25 \times 0.5 \mathrm{~mm}$ orifice array.

\section{A. Wave Diagrams}

It is clear from Figs. 6(a) and 6(d) that more shock reflections occur in the cavity as the steady state pressure is reduced. The time-of-flight method was used to determine the shock locations and times using the plenum cavity transducers. Figure 8(a) shows that the shock and three reflections can be tracked. The reflections occur as the detonation wave pressure blocks the orifice flow, causing stagnation conditions and a rise in the plenum pressure from its steady state value to the supply cylinder value. Once the detonation wave pressure reduces to a low enough point, an expansion enters the fluidic valve and causes all of the products to exit so refueling can begin. During this process, the contact surface between the wave products and fuel is trapped in the cavity. When the pressure is higher, only one reflection can be tracked. This occurs because the expansion wave enters the fluidic valve much earlier. The Mach number of the incident shock also reduces as the plenum pressure is raised. It is about 1.7 and 1.05 for Figs. 8(a) and 8(b), respectively.

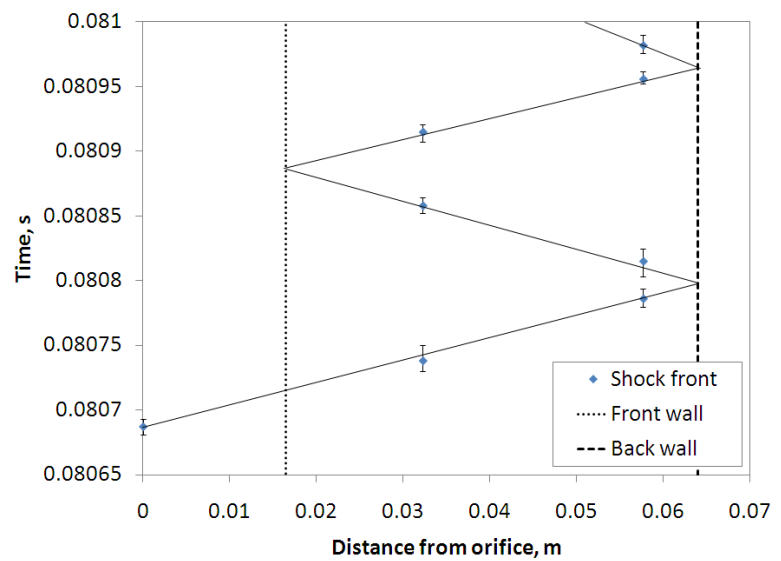

(a) Reflections from Fig. 6(a).

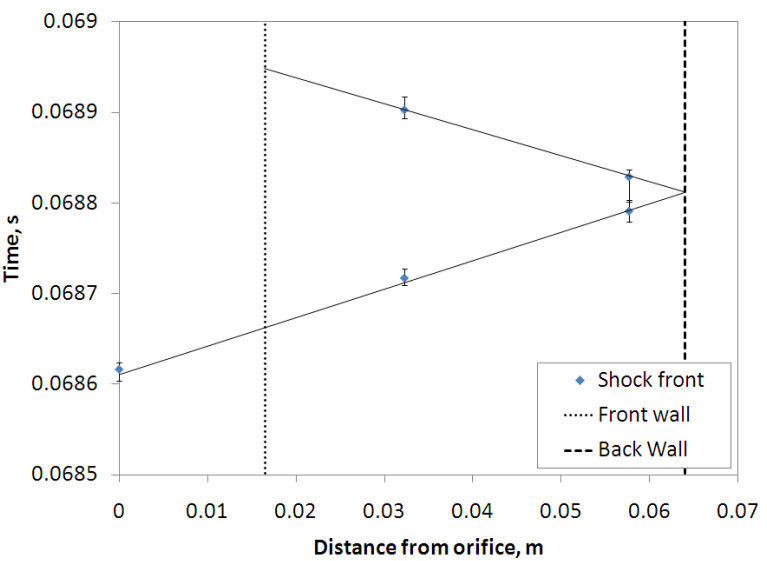

(b) Reflections from Fig. 6(d).

Figure 8. $x-t$ diagrams of shock reflections in the valve cavity.

\section{B. Interruption Time Plots}

For this study, the interruption time is defined as the time between the blast wave front and the point where the plenum pressure returns to its steady state value in the fluidic valve. As was mentioned earlier, this interruption time is not necessarily the backflow time. It is limited in that it cannot be used to predict if 
the steady-state flow is indeed comprised of the detonation products or new propellants since the contact surface position cannot be directly measured. A true backflow time measurement would require either a CFD analysis or optical windows mounted on the walls of the plenum cavity. However, this interruption time can be useful for comparisons between the experimental variables since the true backflow time and interruption time as defined should follow the same overall trends.

Figure 9 shows an example of the graphical methodology used to determine the interruption time and its uncertainty. First, the steady state pressure is determined and graphed across the figure. The plenum pressure rises with the blast wave and then drops back to its initial level. The recorded pressure usually exhibits an oscillatory behavior so the first dashed line indicates the first point in time where the wave pressure reaches its initial value. The solid line indicates where the oscillatory pressure trace appears to be centered on the line of steady state pressure. The distance between the two is the uncertainty, which is mirrored on the graph with a second band. The interruption time result is labeled.

After calculating the interruption time for many experimental conditions, it was found that the results could be placed in a non-dimensional form to

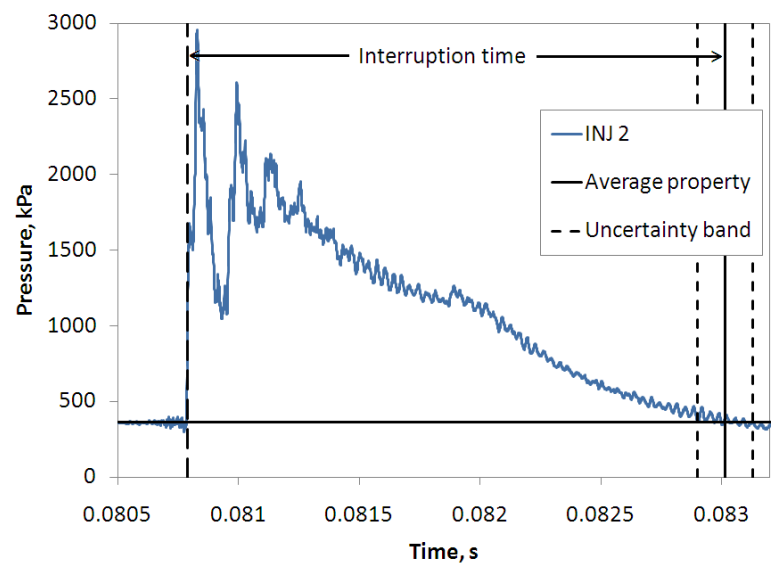

Figure 9. Graphical method for determining the interruption time. show what conditions control the behavior of the fluidic valves. The interruption time may be divided by the characteristic time of the gas dynamics in the main detonation tube to form ${ }^{17}$

$$
\tau=\frac{t_{i n t}}{L / U_{C J}}
$$

In Eq. (1), $L$ is the distance from the ignition source to the fluidic valve orifice. The detonation speed $U_{C J}$ was calculated for each experiment using the time-of-flight method with the transducers mounted along the main tube. Since the steady state injection pressure was one of the main variables during testing, another non-dimensional term was created by dividing it by the pressure ratio of the detonation wave.

$$
\Pi=\frac{\pi_{I}}{\pi_{C J}}=\frac{p_{P} / p_{0}}{p_{C J} / p_{0}}
$$

The cavity and initial pressures are determined experimentally. The CJ wave pressure was estimated with $\mathrm{CEA}^{18}$ using the molar ratio of the mixture in the tube prior to running.

Figure 10 shows the experimental data graphed with these parameters while comparing the orifice geometry and fuel used. A linear trend is apparent between $\tau$ and $\Pi$. Every interruption time measurement follows the linear trend, regardless if the injected gas was argon, oxygen, or hydrogen. The two injector orifices used also follow the trend. Note that uncertainty in the data is relatively high for $2.0<\tau<2.5$ due to the reflection that occurs in the main tube. For a rotating detonation wave engine, the characteristic length $L$ could be replaced with the circumference of the annulus. If so, an optimal RDE fluidic valve mainly scales with detonation wave pressure, injector pressure, and the frequency of the detonation wave.

$$
t_{\text {int }} \propto \frac{1}{f_{R D E} \Pi}
$$

In several computational and analytical studies, a stable RDE operates with a fraction of fuel injector orifices closed off due to the pressure of the detonation wave. ${ }^{9,11}$ This fraction can then be matched with a ratio of the interruption time to the characteristic time of the rotating wave to estimate the required injection pressure. An iterative solution for this design will probably be required. 


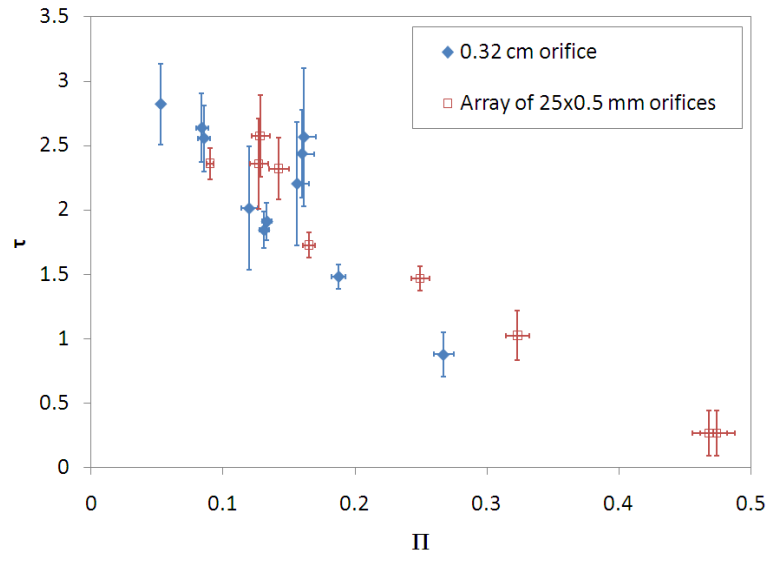

(a) Orifice geometry comparison.

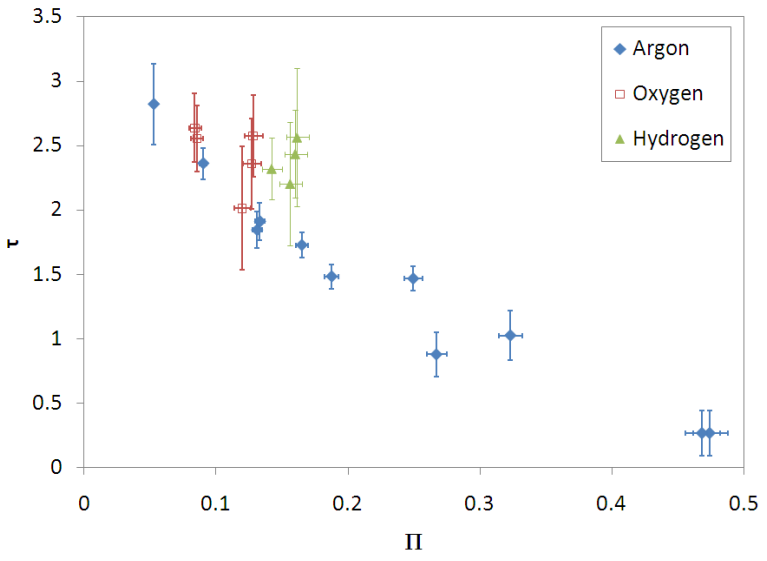

(b) Fuel comparison.

Figure 10. Dimensionless plots of interruption time versus the steady state injection pressure.

\section{Conclusions}

The goal of this study was to understand how fuel injection for high-frequency RDEs or PDEs may be accomplished since electromechanical valves likely cannot operate fast enough. The approach taken centered upon what has been termed a fluidic valve, which incorporates a plenum cavity that injects fuel at a steady state pressure and counteracts the detonation wave pressure to ensure rapid refueling. Different fuels, orifice geometries, and plenum cavity pressures were varied in several parametric studies. Early RDE studies have discussed the possibility of backflow into the injectors from the detonation wave, but the effects and potential solutions have not been well investigated. Pressure transducers were placed in the valve cavity in order to understand the dynamics and interaction with a single-shot detonation wave traveling in a linear tube. An interruption time was defined to measure the time required for the fluidic valve to return to steady-state injection after the detonation front passes the injector orifice. The metric is based solely on pressure readings, and a computational fluid dynamics study may be required to reveal how this time corresponds to when fresh mixture also begins to flow out of the valve again. The points below summarize the fluidic valve results:

- It appears possible to build a fluidic valve that can return to refueling at steady-state conditions between detonation wave fronts. Although the single-shot experiments cannot replicate operating frequencies that might be realized in an RDE, the fluidic valve was able to interact with characteristic times of the detonation tube that correspond an operating frequency of over $500 \mathrm{~Hz}$. There is no current reason to believe that the orifice and cavity geometry cannot be scaled to integrate with an RDE.

- Although different fuels and orifice geometries were used, the non-dimensional interruption time plots show that this fluidic valve predominantly scales with a characteristic time of the tube (or annulus) and the ratio of the injection pressure to the detonation wave pressure.

- Injectors with small sets of orifices with diameters in the $0.5 \mathrm{~mm}$ range have been used previously with RDEs. Larger diameter orifices could be employed (although it is recognized that small orifices may still be more suitable for mixing).

\section{Acknowledgments}

This work was supported by HyPerComp, Inc. through a DARPA SBIR subcontract. The authors would like to thank Dr. Ramakanth Munipalli for several productive conversations as well as Nathan L. Dunn and Steven McClendon for assistance with the experiments. The views, opinions, and/or findings contained in this article/presentation are those of the author/presenter and should not be interpreted as representing the official views or policies, either expressed or implied, of the Defense Advanced Research Projects Agency or the Department of Defense. 


\section{References}

${ }^{1}$ Kailasanath, K., "Recent Developments in the Research on Pulse Detonation Engines," AIAA Journal, Vol. 41, No. 2, 2003, pp. 145-159.

${ }^{2}$ Roy, G. D., Frolov, S. M., Borisov, A. A., and Netzer, D. W., "Pulse Detonation Propulsion: Challenges, Current Status, and Future Perspective," Progress in Energy and Combustion Science, Vol. 30, No. 6, 2004, pp. 545-672.

${ }^{3}$ Bykovskii, F. A., Zhdan, S. A., and Vedernikov, E. F., "Continuous Spin Detonations," Journal of Propulsion and Power, Vol. 22, No. 6, 2006, pp. 1204-1216.

${ }^{4}$ Canteins, G., A Study of Continuous Rotating Detonation with Application to Propulsion, Doctoral dissertation, University of Poitiers, Poitiers, France, 2006.

${ }^{5}$ Braun, E. M., Dunn, N. L., and Lu, F. K., "Testing of a Continuous Detonation Wave Engine with Swirled Injection," 48th AIAA Aerospace Sciences Meeting, AIAA Paper 2010-146, Orlando FL, 2010.

${ }^{6}$ Hishida, M., Fujiwara, T., and Wolanski, P., "Fundamentals of Rotating Detonations," Shock Waves, Vol. 19, No. 1, 2009, pp. 1-10.

${ }^{7}$ Kindracki, J., Wolanski, P., and Gut, Z., "Experimental Research on the Rotating Detonation in Gaseous Fuels-Oxygen Mixtures," Shock Waves, Vol. 21, No. 2, 2011, pp. 75-84.

${ }^{8}$ Zhdan, S. A., "Mathematical Model of Continuous Detonation in an Annular Combustor with a Supersonic Flow Velocity," Combustion, Explosion, and Shock Waves, Vol. 44, No. 6, 2008, pp. 690-697.

${ }^{9}$ Yi, T.-H., Lou, J., Turangan, C., Choi, J.-Y., and Wolanski, P., "Propulsive Performance of a Continuously Rotating Detonation Engine," Journal of Propulsion and Power, Vol. 27, No. 1, 2011, pp. 171-181.

${ }^{10}$ Schwer, D. A. and Kailasanath, K., "Numerical Investigation of Rotating Detonation Engines," 46th AIAA/ASME/SAE/ASEE Joint Propulsion Conference \& Exhibit, AIAA Paper 2010-6880, Nashville TN, 2010.

${ }^{11}$ Braun, E. M., Lu, F. K., Wilson, D. R., and Camberos, J. A., "Airbreathing Rotating Detonation Wave Engine Cycle Analysis," 46th AIAA/ASME/SAE/ASEE Joint Propulsion Conference E Exhibit, AIAA Paper 2010-7039, Nashville TN, 2010.

${ }^{12}$ Nicholls, J. A. and Cullen, R. E., "The Feasibility of a Rotating Detonation Wave Rocket Motor," Tech. Rep. RPL-TDR64-113, The University of Michigan, Ann Arbor, MI, April 1964.

${ }^{13}$ Bykovskii, F. A. and Vedernikov, E. F., "Self-Sustaining Pulsating Detonation of a Gas-Mixture Flow," Combustion, Explosion, and Shock Waves, Vol. 32, No. 4, 1996, pp. 442-448.

${ }^{14}$ Sutton, G. P. and Biblarz, O. B., Rocket Propulsion Elements, John Wiley \& Sons, New York, 2001.

${ }^{15}$ Bykovskii, F. A. and Mitrofanov, V. V., "Detonation Combustion of a Gas Mixture in a Cylindrical Chamber," Combustion, Explosion, and Shock Waves, Vol. 16, No. 5, 1981, pp. 570-578.

${ }^{16}$ Bykovskii, F. A., "Thermal Fluxes in Combustion Chamber Walls in the Detonation and Turbulent Combustion Modes," Combustion, Explosion, and Shock Waves, Vol. 27, No. 1, 1991, pp. 66-71.

${ }^{17}$ Endo, T., Kasahara, J., Matsuo, A., Inaba, K., Sato, S., and Fujiwara, T., "Pressure History at the Thrust Wall of a Simplified Pulse Detonation Engine," AIAA Journal, Vol. 42, No. 9, 2004, pp. 1921-1930.

${ }^{18}$ Gordon, S. and McBride, B. J., "Computer Program for Calculation of Complex Chemical Equilibrium Compositions, Rocket Performance, Incident and Reflected Shocks, and Chapman-Jouguet Detonations," Tech. Rep. NASA SP-273, 1971. 